\title{
Hybrid meshless displacement discontinuity method (MDDM) in fracture mechanics: static and dynamic
}

\author{
J. Li ${ }^{1}$, J. Sladek ${ }^{2}$, V. Sladek ${ }^{2}$ and P.H. Wen ${ }^{3 *}$ \\ ${ }^{1}$ School of Mathematics and Statistics, Changsha University of Science and Technology, China \\ ${ }^{2}$ Institute of Construction and Architecture, Slovak Academy of Sciences, 84503 Bratislava, Slovakia \\ ${ }^{3}$ School of Engineering and Materials Science, Queen Mary, University of London, London, UK, E1 4NS
}

\begin{abstract}
This paper investigated a hybrid Meshless Displacement Discontinuity Method (MDDM) for a cracked plate subjected to static and dynamic loadings. The purpose of MDDM is to model displacement discontinuity on a cracked surface by the displacement discontinuity method in an infinite plate. This was achieved by considering a meshless approach, the equilibrium equations, and the boundary conditions for a domain with an irregular nodes distribution. Also, by imposing the principle of superposition, accurate and convergent solutions can be obtained. In this paper, the static and dynamic stress intensity factors, and the crack growth for different initial crack length and crack slant angles are investigated. The Laplace transform method is applied to deal with dynamic problems and the time-dependent values are obtained by the Durbin inversion technique. Validations of the presented technique are demonstrated by four numerical examples of plates with a central embedded crack.
\end{abstract}

Key words: Boundary element method, meshless method, stress intensity factors, static and dynamic loadings, crack growth.

*Corresponding: p.h.wen@qmul.ac.uk (P.H.Wen) 


\section{Introduction}

It is well known that the numerical simulation of crack-growth processes is mature as many numerical strategies including the finite element method (FEM) have been developed. As a general numerical tool, FEM has been developed for crack propagation simulation in solid structures $[1,2,3]$. The early attempt to model crack growth in mixed-mode conditions was reported by Gallagher [4], Rice and Tracey [5], Shephard et al [6] and Rice [7]. Recently, the extended finite element method (XFEM) $[8,9,10]$ was proposed for fatigue/ fracture analysis in nonhomogeneous materials. In spite of the great success of general boundary value problems, the new and advanced computational methods are still required due to the need for computational accuracy and efficiency, and structure complexity. As the discontinuities of the stress/strain by FEM between elements affect the accuracy significantly, the boundary element method (BEM) is able to achieve high accurate solutions. The two main advantages of BEM is the reduction of the spatial dimensions by at least one and the high accuracy is achieved especially if the domain of interest is infinite or semi-infinite. Early investigations of mixedmode crack growth conditions by BEM were reported by Ingraffea et al [11] and Grestle [12] for two- and three-dimensional problems with the multi-region technique. Cen and Maier [13] applied BEM to simulate crack growth in concrete structures. In the 1990's, the Dual Boundary Element Method (DBEM) with a single region technique for the crack growth analysis was demonstrated by Portela et al [14] for two-dimensional and by Mi and Aliabadi [15,16] for three-dimensional problems. One of the advantages of DBEM is that the crack extension procedure can be modelled easily by new elements. For DBEM applications in crack mechanics, a general review was given by Aliabadi [17]. Apart from DBEM, the indirect boundary element method is another accurate method formulated with the principle of superposition including the Fictitious Load Method (FLM) and the Displacement Discontinuity Method (DDM). This was reported by Crouch [18] in the "Boundary Element Methods in Solid Mechanics". The DDM was extended to static/dynamic 2D/3D fracture mechanics by Wen et al in $[19,20,21,22,23]$.

How to interpolate a variable accurately using the values of irregular node arrangement in a domain is a fundamental task in meshless methods. The multiquadric Radial Basis Function (RBF) $R(r)=\sqrt{c^{2}+r^{2}}$ was studied by Hardy [24] in 1971 for topographical surfaces, and this can be considered as the first development for meshless method. Similar to the Moving Least 
Square (MLS) algorithm, the compact support RBF has been explicitly constructed to multivariate surface reconstruction. Later Belyschko et al [25] developed the element-free Galerkin method (EFGM) based on accurate interpolation methods including the MLS and RBF respectively. Pathak et al [26] developed and utilised the enriched EFGM method to investigate fatigue problems in homogeneous and bi-material interfacial cracks structures. Jameel and Harmain [27] also investigated fatigue crack growth of material discontinuities using the EFG method, and Muthu et al [28] also investigated the impact of T-stress on crack propagation using a variant of the EFG method. In addition, the local support domain technique provides a form of theoretical basis for large scale problems, see Hon et al [29]. Atluri et al [30] reported a series of Meshless Local Petrov-Galerkin formulations (MLPGs) for general partial differential equations with MLS approximation from the past two decades. The local boundary integral equation with the MLS and RBF was reported by Sladek et al [31,32] to deal with fracture problems in anisotropic non-homogeneous media. With enriched RBF at the crack tip, Wen and Aliabadi [33] demonstrated the application of meshless method to fracture problems with functionally graded materials. However, a drawback of the meshless method is the accuracy and convergence, precisely to the modelling of singular stresses at the crack tip by using the MLS and RBF interpolations. In the present paper, we aim to develop a hybrid method with the DDM and mshless method in order to take advantages of both the boundary element method (high accuracy) and the meshless method (body force terms). The advantages and disadvantages for meshless strong form method and displacement discontinuity method are listed in Table 1.

The time-dependent values of displacement, stress and stress intensity factor are obtained from an inverse transform of the corresponding Laplace-transformed quantities. Great attention has been paid to the numerical inversion of the Laplace transformation. General introduction of the Laplace invers was given by $\mathrm{Fu}$ et al $[34,35,36]$. In this paper, the formulations for crack problem are coupled with distributed dislocation on the crack surface (discontinuous field in infinite plate) and meshless solution (continuous field) to deal with fracture problems. The crack growth is simulated simply by adding a new crack segments ahead of the crack tip. The Laplace transform technique is applied to deal with dynamic problems by using static Kelvin solution of displacement discontinuity for 2D elasto-dynamic problems and the Durbin inversion method [37] is used to obtain the time-dependent solution. Numerical results of cracked rectangular plate and cracked circular plate are presented to illustrate the applicability 
and degree of accuracy with the MDDM. Comparisons with analytical and BEM results show a good agreement.

Table 1 The advantages and disadvantages of meshless and DDM methods

\begin{tabular}{|c|c|c|}
\hline Method & Advantages & Disadvantages \\
\hline BEM/DDM & $\begin{array}{l}\text { Dimension reduction, excellent } \\
\text { for crack modelling, infinite } \\
\text { domain, static/dynamic loads, } \\
\text { high accuracy, high convergent, } \\
\text { easy to code; Computational } \\
\text { effort is much less than other } \\
\text { methods in solving linear } \\
\text { algebraic equations. }\end{array}$ & $\begin{array}{l}\text { Fundamental solutions, not } \\
\text { available for material and } \\
\text { geometry nonlinear problem. }\end{array}$ \\
\hline $\begin{array}{c}\text { Meshless strong } \\
\text { form methods }\end{array}$ & $\begin{array}{l}\text { True mesh free, computational } \\
\text { efficient to treat nonlinear } \\
\text { problem, good convergent rate, } \\
\text { easy to code; no fundamental } \\
\text { solutions; More computational } \\
\text { effort used. }\end{array}$ & $\begin{array}{l}\text { Free parameters, unstable, } \\
\text { convergence issue, not accuracy } \\
\text { for crack problem. }\end{array}$ \\
\hline
\end{tabular}

\section{Displacement discontinuity method}

The displacement discontinuity method is attributed to one of the indirect boundary element techniques. Using the displacement discontinuity method, a high level of accuracy and rapid convergence is obtained when applied in fracture analysis to determine the stress intensity factors. Consider a concentrated force acting at point $\mathrm{A}(x, y)$ as shown in Figure 1 in an infinite plate, the fundamental solution of the stress tensor is given, for 2D plane strain problem [17], by

$$
S_{i j k}^{*}(\mathbf{X}-\mathbf{x})=\frac{1}{4 \pi(1-v) r}\left[(1-2 v)\left(r_{, k} \delta_{i j}-r_{, j} \delta_{i k}-r_{, i} \delta_{j k}\right)-2 r_{, i} r_{, j} r_{, k}\right]
$$


where $r=|\mathbf{X}-\mathbf{x}|, r_{, i}=\left(X_{i}-x_{i}\right) / r, \mathbf{X}$ is the coordinate of considered point and $v$ is the Poisson ratio, subscript $k$ denotes the direction of concentrated force. Consider an opening mode (I) displacement discontinuity at the origin as shown in Figure 1, i.e.

$$
u_{2}^{+}-u_{2}^{-}=\delta(x, 0), u_{1}^{+}-u_{1}^{-}=0
$$

where the superscript + and - indicate the upper and lower semi-infinite plates. The displacement field can be obtained by the reciprocal theory

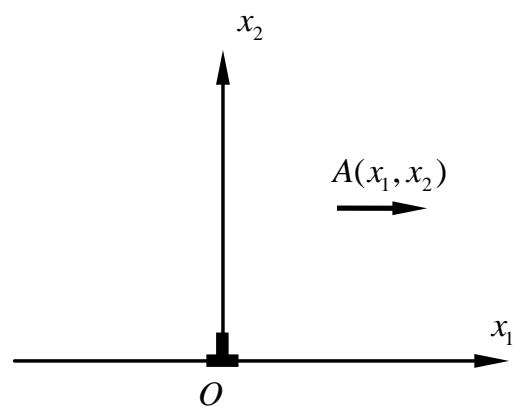

Figure 1. Displacement discontinuity at the origin.

$$
u_{k 2}^{*}(\mathbf{x})=S_{2 k 2}^{*}(-\mathbf{x})=\frac{-1}{4 \pi(1-v) r}\left[(1-2 v)\left(x_{k}-x_{2} \delta_{2 k}-x_{2} \delta_{2 k}\right) / r-2 x_{2}^{2} x_{k} / r^{3}\right]
$$

where subscript $k(=1,2)$ indicates the direction of displacement. Consider a shear mode (II) displacement discontinuity at the origin, i.e.

$$
u_{1}^{+}-u_{1}^{-}=\delta(x, 0), u_{2}^{+}-u_{2}^{-}=0 .
$$

Then, the displacements can be obtained as

$$
u_{k 1}^{*}(\mathbf{x})=S_{12 k}^{*}(-\mathbf{x})=\frac{1}{4 \pi(1-v) r}\left[(1-2 v)\left(x_{2} \delta_{1 k}+x_{1} \delta_{2 k}\right) / r+2 x_{1} x_{2} x_{k} / r^{3}\right]
$$

By Hook's law, the fundamental solution of stress tensor $\sigma_{i j k}^{*}(\mathbf{x})$ deriving from the fundamental displacement above is given by

$$
\sigma_{i j k}^{*}(\mathbf{x})=C_{i j n l} \varepsilon_{n l k}^{*}(\mathbf{x})
$$

where $\varepsilon_{n l k}^{*}=\left(u_{k n, l}^{*}+u_{k l, n}^{*}\right) / 2$, and $C_{i j n l}$ denotes the elasticity tensor. For a homogeneous isotropic solid, we have 


$$
C_{i j n l}=\lambda \delta_{i j} \delta_{n l}+\mu\left(\delta_{i n} \delta_{j l}+\delta_{i l} \delta_{j n}\right)
$$

where $\lambda$ is the Lame's constant and $\mu$ is the shear modulus. Consider a constant displacement discontinuity distributed on a straight line shown in Figure 2(a), the displacement and stress fields are obtained by the following integrations

$$
u_{i}^{(l)}(\mathbf{x})=\int_{-\Delta^{(l)}}^{\Delta^{(l)}} u_{i k}^{*}\left(x_{1}-t, x_{2}\right) \psi_{k} d t \quad \text { and } \quad \sigma_{i j}^{(l)}(\mathbf{x})=\int_{-\Delta^{(l)}}^{\Delta^{(l)}} \sigma_{i j k}^{*}\left(x_{1}-t, x_{2}\right) \psi_{k}^{(l)} d t
$$

where $\Delta^{(l)}$ is half-length of the constant element $l, \psi_{k}^{(l)}$ is the displacement discontinuity of different modes ( $k=1$ signify the shear mode and $k=2$ the opening mode). Integrating (8) results [18]

$$
u_{i}^{(l)}(\mathbf{x})=U_{i k}^{(l)}(\mathbf{x}) \psi_{k}^{(l)} \quad \text { and } \quad \sigma_{i j}^{(l)}(\mathbf{x})=T_{i j k}^{(l)}(\mathbf{x}) \psi_{k}^{(l)}
$$

where

$$
\begin{aligned}
& U_{11}^{(l)}(\mathbf{x})=-2(1-v) f_{, 2}+x_{2} f_{, 11}, \quad U_{21}^{(l)}(\mathbf{x})=-(1-2 v) f_{, 1}+x_{2} f_{, 12}, \\
& U_{12}^{(l)}(\mathbf{x})=(1-2 v) f_{, 1}+x_{2} f_{, 12}, \quad U_{22}^{(l)}(\mathbf{x})=-2(1-v) f_{, 2}+x_{2} f_{, 22},
\end{aligned}
$$

for displacement and

$$
\begin{aligned}
& T_{111}^{(l)}(\mathbf{x})=2 \mu\left(-2 f_{, 12}-x_{2} f_{, 122}\right), T_{221}^{(l)}(\mathbf{x})=2 \mu x_{2} f_{, 122}, T_{121}^{(l)}(\mathbf{x})=2 \mu\left(-f_{, 22}-x_{2} f_{, 222}\right), \\
& T_{112}^{(l)}(\mathbf{x})=2 \mu\left(-f_{, 22}-x_{2} f_{, 222}\right), T_{222}^{(l)}(\mathbf{x})=2 \mu\left(-f_{, 22}+x_{2} f_{, 222}\right), T_{122}^{(l)}(\mathbf{x})=2 \mu x_{2} f_{, 122} .
\end{aligned}
$$

in which

$$
\begin{aligned}
f\left(x_{1}, x_{2}\right)= & -\frac{1}{4 \pi(1-v)}\left[x_{2}\left(\arctan \left(\frac{x_{2}}{x_{1}-\Delta}\right)-\arctan \left(\frac{x_{2}}{x_{1}+\Delta}\right)\right)\right. \\
& \left.-\left(x_{1}-\Delta\right) \ln \sqrt{\left(x_{1}-\Delta\right)^{2}+x_{2}^{2}}+\left(x_{1}+\Delta\right) \ln \sqrt{\left(x_{1}+\Delta\right)^{2}+x_{2}^{2}}\right]
\end{aligned}
$$

Different orders of the partial derivative function $f\left(x_{1}, x_{2}\right)$ used in the DDM are given in Appendix A. The normal and tangential components of the displacements and tractions at the centre of segment $m$ caused by displacement continuities $\psi_{k}^{(l)}$ of element $l$ shown in Figure 2(b) can be determined by

$$
\begin{aligned}
& u^{n}\left(\mathbf{x}^{(l m)}\right)=\left[U_{1 k}\left(\mathbf{x}^{(l m)}\right) \operatorname{co}\left(\theta^{(m)}-\theta^{(l)}\right)+U_{2 k}\left(\mathbf{x}^{(I m)}\right) \sin \theta^{(m)}\left(-\theta^{(l)}\right)\right] \psi_{k}^{(l)}=U_{k}^{n(l m)} \psi_{k}^{(l)}, \\
& u^{\tau}\left(\mathbf{x}^{(l m)}\right)=\left[-U_{1 k}\left(\mathbf{x}^{(l m)}\right) \sin \theta^{(m)}\left(-\theta^{(l)}\right)+U_{2 k}\left(\mathbf{x}^{(l m)}\right) \operatorname{co}\left(\theta^{(m)}-\theta^{(l)}\right)\right] \psi_{k}^{(l)}=U_{k}^{\tau(l m)} \psi_{k}^{(l)},
\end{aligned}
$$




$$
\begin{aligned}
t^{n}\left(\mathbf{x}^{(I m)}\right)= & {\left[T_{11 k}\left(\mathbf{x}^{(I m)}\right) \operatorname{co}^{2}\left(\theta \mathrm{s}^{(m)}-\theta^{(l)}\right)+T_{22 k}\left(\mathbf{x}^{(l m)}\right) \sin ^{2}\left(\theta^{(m)}-\theta^{(l)}\right)\right.} \\
& \left.+T_{12 k}\left(\mathbf{x}^{(l m)}\right) \sin 2\left(\theta^{(m)}-\theta^{(l)}\right)\right] \psi_{k}^{(l)}=T_{k}^{n(l m)} \psi_{k}^{(l)}, \\
t^{\tau}\left(\mathbf{x}^{(l m)}\right)= & {\left[\frac{T_{11 k}\left(\mathbf{x}^{(l m)}\right)-T_{22 k}\left(\mathbf{x}^{(l m)}\right)}{2} \sin 2\left(\theta^{(m)}-\theta^{(l)}\right)+T_{12 k}\left(\mathbf{x}^{(l m)}\right) \cos 2\left(\theta^{(m)}-\theta^{(l)}\right)\right] \psi_{k}^{(l)} } \\
= & T_{k}^{\tau(l m)} \psi_{k}^{(l)}
\end{aligned}
$$

where $\mathbf{x}^{(l m)}=\left(x_{1}^{(l m)}, x_{2}^{(l m)}\right), x_{1}^{(I m)}=\left(x_{1}^{(m)}-x_{1}^{(l)}\right)$ co $\theta^{(l)}+\left(x_{2}^{(m)}-x_{2}^{(l)}\right) \sin \theta^{(l)}, x_{2}^{(l m)}=-\left(x_{1}^{(m)}-x_{1}^{(l)}\right) \times$ $\sin \theta^{(l)}+\left(x_{2}^{(m)}-x_{2}^{(l)}\right) \cos \theta^{(l)}, U_{k}^{n(l m)}, U_{k}^{\tau(l m)}, T_{k}^{n(I m)}$ and $T_{k}^{\tau(l m)}$ are all named as influence coefficients. Considering a curved crack with $N_{c}$ segments in infinite plate shown in Figure 2(b) with the following traction boundary conditions

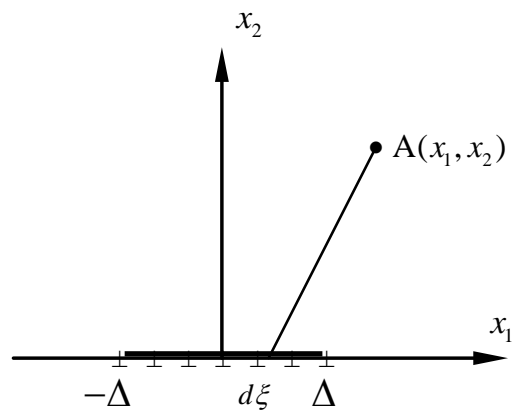

(a)

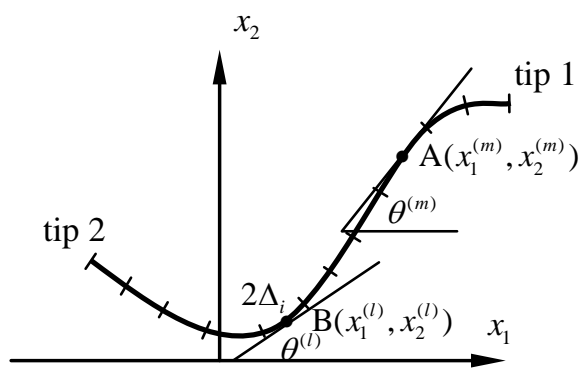

(b)

Figure 2. Constant displacement discontinuity: (a) A displacement discontinuity element in the local coordinate system; (b) Influence between two segments $l$ and $m$.

$$
\sum_{l=1}^{N_{c}} T_{k}^{n(l m)} \psi_{k}^{(l)}=\bar{t}^{n}\left(\mathbf{x}^{(m)}\right) \quad \text { and } \sum_{l=1}^{N_{c}} T_{k}^{\tau(l m)} \psi_{k}^{(l)}=\bar{t}^{\tau}\left(\mathbf{x}^{(m)}\right) \quad\left(m=1,2, \ldots, N_{c}\right)
$$

where $\bar{t}^{n}\left(\mathbf{x}^{(m)}\right)$ and $\bar{t}_{\tau}^{m}\left(\mathbf{x}^{(m)}\right)$ denote the prescribed normal and tangential tractions on element $m$, respectively, and $\psi_{k}^{(l)}(k=1,2)$ are unknown displacement discontinuities for each segment. By solving a set of linear algebraic equations in Eq.(14), $2 N_{c}$ unknown displacement discontinuities $\psi_{k}^{(l)}$ can be obtained.

\section{Meshless approach with radial basis function}


Consider a domain $\Omega$ surrounded by a boundary $\Gamma$ and a sub-domain $\Omega_{s}$ centred at point $\boldsymbol{\eta}$ $\left(x_{1}^{(m)}, x_{2}^{(m)}\right)$ shown in Figure 3 . With scattered nodes $\xi_{p}=\left(x_{1}^{(p)}, x_{2}^{(p)}\right)\left(p=1,2, \ldots, L_{m}\right)$ in the sub-domain, function $u$ can be approximated as

$$
u(\boldsymbol{\eta})=\sum_{p=1}^{L_{m}} R_{p}(\boldsymbol{\eta}) a_{p}+\sum_{q=1}^{Q} P_{q}(\boldsymbol{\eta}) b_{q}=\mathbf{R}(\boldsymbol{\eta}) \mathbf{a}+\mathbf{P}(\boldsymbol{\eta}) \mathbf{b}
$$

where $L_{m}$ is the number of scattered points in the sub-domain $\Omega_{s}$, $\left.\mathbf{R} \mathfrak{\eta})=\left\{R_{1} \mathfrak{\eta}\right), R_{2} \mathfrak{\eta}\right)$,. . $\left.\left.R_{\mathrm{L}_{m}} \mathfrak{h}\right)\right\}$ is the vector of the Radial Basis Function (RBF) associated nodal values and centred at the point $\boldsymbol{\eta},\left\{a_{p}\right\}_{p=1}^{L_{m}}$ are unknown coefficients, $Q$ is the number of polynomial basis functions which can be chosen based on the reproduction requirement and $\left\{b_{q}\right\}_{q=1}^{Q}$ are unknown coefficients for the polynomial basis $\left\{p_{q}(\boldsymbol{\eta})\right\}_{q=1}^{Q}$. We noticed that the contribution of the polynomial basis by computational tests can be ignored. In general, a meshless method utilizes an interpolation to represent the trial function with the values (or the fictitious values) of the variable at scattered nodes in a local area. The moving least-squares (MLS) approximation, the Kringing interpolation and the radial basis function interpolation have slight difference in accuracy. However, the radial basis function interpolation is the simplest with the lesser computational effort. The radial basis function is selected as multiquadrics [24] in this paper as

$$
R_{p}(\mathbf{x})=\sqrt{c^{2}+\left(x_{1}-x_{1}^{(p)}\right)^{2}+\left(x_{2}-x_{2}^{(p)}\right)^{2}}
$$

where $c$ is a free parameter. Therefore, we have

$$
u(\boldsymbol{\eta})=\mathbf{R}(\boldsymbol{\eta}) \mathbf{R}_{0}^{-1} \mathbf{u}=\sum_{p=1}^{L_{m}} \phi_{p}(\boldsymbol{\eta}) u^{(p)}
$$

where $\mathbf{u}=\left\{u^{(1)}, u^{(2)}, \ldots, u^{\left(L_{m}\right)}\right\}^{\mathrm{T}}, u^{(p)}$ represents the nodal value of the scattered node $p$ in the subdomain, $\phi_{p}(\boldsymbol{\eta})$ is defined as the shape function and the matrix 


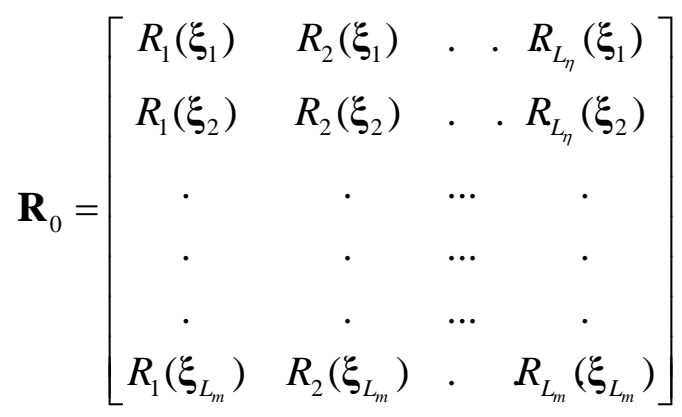

For 2D elasticity, the governing equations are given, for plane strain case, as

$$
\begin{aligned}
& \frac{2 \mu(1-v)}{(1-2 v)} \frac{\partial^{2} u_{1}}{\partial x_{1}^{2}}+\mu \frac{\partial^{2} u_{1}}{\partial x_{2}^{2}}+\frac{\mu}{1-2 v} \frac{\partial^{2} u_{2}}{\partial x_{1} \partial x_{2}}+b_{1}=0 \\
& \frac{\mu}{1-2 v} \frac{\partial^{2} u_{1}}{\partial x_{1} \partial x_{2}}+\frac{2 \mu(1-v)}{(1-2 v)} \frac{\partial^{2} u_{2}}{\partial x_{2}^{2}}+\mu \frac{\partial^{2} u_{2}}{\partial x_{1}^{2}}+b_{2}=0
\end{aligned}
$$

where $b_{k}$ are body forces. With the RBF interpolation by Eq.(16), one has a set of linear algebraic equation from Eq.(18)

$$
\begin{aligned}
& \sum_{p=1}^{L_{m}}\left(\frac{2 \mu(1-v)}{(1-2 v)} \phi_{p, 11}+\mu \phi_{p, 22}\right) u_{1}^{(p)}+\sum_{p=1}^{L_{m}}\left(\frac{\mu}{(1-2 v)} \phi_{p, 12}\right) u_{2}^{(p)}=-b_{1} \\
& \sum_{p=1}^{L_{m}}\left(\frac{\mu}{(1-2 v)} \phi_{p, 12}\right) u_{1}^{(p)}+\sum_{p=1}^{L_{m}}\left(\frac{2 \mu(1-v)}{(1-2 v)} \phi_{p, 22}+\mu \phi_{p, 11}\right) u_{2}^{(p)}=-b_{2}
\end{aligned}
$$

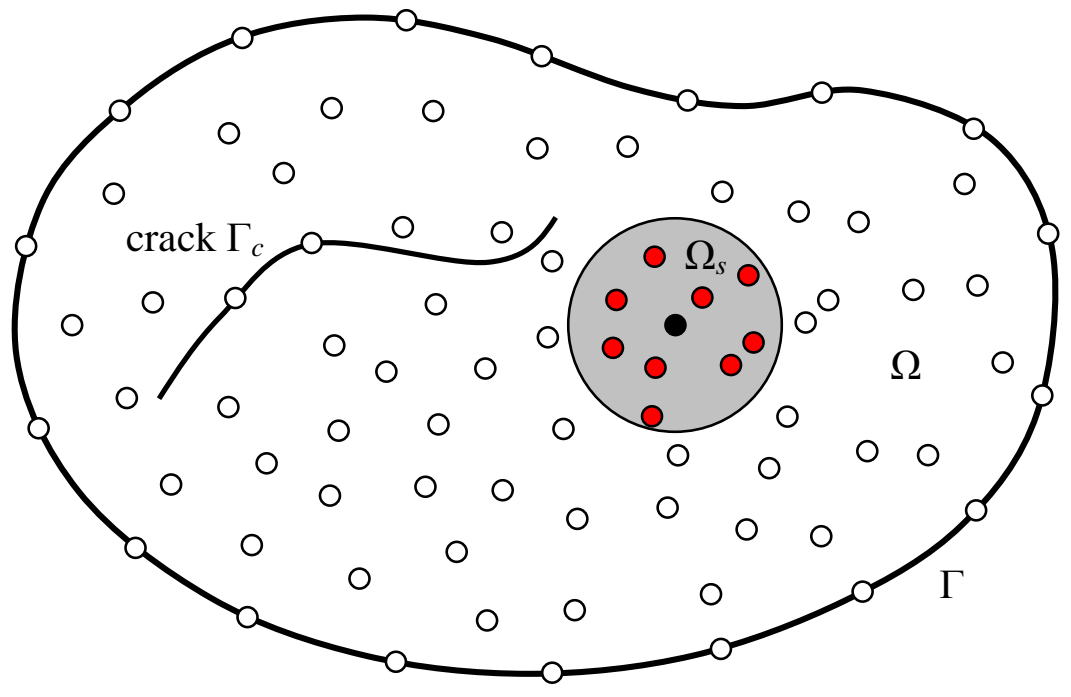

Figure 3. Support-domain $\Omega_{s}$ centred at field point $\boldsymbol{\eta}$ : o nodes in domain; collocation point; o nodes in support domain. 
for collocation points $\boldsymbol{\eta}_{m}, m=1,2, \ldots, N_{m}, N_{m}$ is the number of scattered nodes in the domain $\Omega$. For each boundary collocation point, we have boundary conditions in the normal and tangential $(n, \tau)$ directions as

(1) Displacement boundary condition

$$
u^{n}\left(\boldsymbol{\eta}_{m}\right)=\left.u_{i} n_{i}\right|_{\left(\boldsymbol{\eta}_{m}\right)}=\bar{u}^{n}\left(\boldsymbol{\eta}_{m}\right), u^{\tau}\left(\boldsymbol{\eta}_{m}\right)=\left.u_{i} \tau_{i}\right|_{\left(\boldsymbol{\eta}_{m}\right)}=\bar{u}^{\tau}\left(\boldsymbol{\eta}_{m}\right)
$$

in which $\mathbf{n}=\left(n_{1}, n_{2}\right)$ is the outward unit normal vector to the boundary, $\tau_{1}=-n_{2}$ and $. \tau_{2}=n_{1}$.

(2) Traction boundary conditions

$$
\left.\left(\sigma_{11} n_{1}^{2}+\sigma_{22} n_{2}^{2}+2 \sigma_{12} n_{1} n_{2}\right)\right|_{\left(\boldsymbol{\eta}_{m}\right)}=\bar{t}^{n}\left(\boldsymbol{\eta}_{m}\right),\left.\left[\left(\sigma_{11}-\sigma_{22}\right) n_{1} n_{2}+\sigma_{12}\left(n_{1}^{2}-n_{2}^{2}\right)\right]\right|_{\left(\boldsymbol{\eta}_{m}\right)}=\bar{t}^{\tau}\left(\boldsymbol{\eta}_{m}\right)
$$

where the stress components are from Hook's law

$$
\begin{aligned}
& \sigma_{11}\left(\boldsymbol{\eta}_{m}\right)=\sum_{p=1}^{L_{m}}\left(\frac{2 \mu(1-v)}{(1-2 v)} \phi_{p, 1} u_{1}^{(p)}+\frac{2 v \mu}{(1-2 v)} \phi_{p, 2} u_{2}^{(p)}\right) \\
& \sigma_{22}\left(\boldsymbol{\eta}_{m}\right)=\sum_{p=1}^{L_{m}}\left(\frac{2 v \mu}{(1-2 v)} \phi_{p, 1} u_{1}^{(p)}+\frac{2 \mu(1-v)}{(1-2 v)} \phi_{p, 2} u_{2}^{(p)}\right) \\
& \sigma_{12}\left(\boldsymbol{\eta}_{m}\right)=\mu \sum_{p=1}^{L_{m}}\left(\phi_{l, 2} u_{1}^{(p)}+\phi_{l, 1} u_{2}^{(p)}\right)
\end{aligned}
$$

Therefore, $2 N_{m}$ the unknown nodal displacements can be obtained by solving a set of linear equations from Eqs (19-21). For a 2D dynamic problem, the governing equations become

$$
\begin{aligned}
& \frac{2 \mu(1-v)}{(1-2 v)} \frac{\partial^{2} u_{1}}{\partial x_{1}^{2}}+\mu \frac{\partial^{2} u_{1}}{\partial x_{2}^{2}}+\frac{\mu}{1-2 v} \frac{\partial^{2} u_{2}}{\partial x_{1} \partial x_{2}}+b_{1}=\rho \frac{\partial^{2} u_{1}}{\partial t^{2}} \\
& \frac{\mu}{1-2 v} \frac{\partial^{2} u_{1}}{\partial x_{1} \partial x_{2}}+\frac{2 \mu(1-v)}{(1-2 v)} \frac{\partial^{2} u_{2}}{\partial x_{2}^{2}}+\mu \frac{\partial^{2} u_{2}}{\partial x_{1}^{2}}+b_{2}=\rho \frac{\partial^{2} u_{2}}{\partial t^{2}}
\end{aligned}
$$

where $t$ is the time and $\rho$ is the mass density of the plate. Let the body forces be zero and the speed of longitudinal wave $c_{1}=[2 \mu(1-v) / \rho(1-2 v)]^{1 / 2}$, Eqs (23a,23b) become

$$
\frac{\partial^{2} u_{1}}{\partial x_{1}^{2}}+\frac{(1-2 v)}{2(1-v)} \frac{\partial^{2} u_{1}}{\partial x_{2}^{2}}+\frac{1}{2(1-v)} \frac{\partial^{2} u_{2}}{\partial x_{1} \partial x_{2}}=\frac{1}{c_{1}^{2}} \frac{\partial^{2} u_{1}}{\partial t^{2}}
$$




$$
\frac{1}{2(1-v)} \frac{\partial^{2} u_{1}}{\partial x_{1} \partial x_{2}}+\frac{\partial^{2} u_{2}}{\partial x_{2}^{2}}+\frac{(1-2 v)}{2(1-v)} \frac{\partial^{2} u_{2}}{\partial x_{1}^{2}}=\frac{1}{c_{1}^{2}} \frac{\partial^{2} u_{2}}{\partial t^{2}}
$$

To eliminate time $t$ in Eq.(23), the Laplace transformation

$$
\mathcal{L}[f(\mathbf{x}, t)]=\tilde{f}(\mathbf{x}, p)=\int_{0}^{\infty} f(\mathbf{x}, t) e^{-p t} d
$$

is applied to this system of governing equations and one has

$$
\begin{aligned}
& \frac{\partial^{2} \tilde{u}_{1}}{\partial x_{1}^{2}}+\frac{(1-2 v)}{2(1-v)} \frac{\partial^{2} \tilde{u}_{1}}{\partial x_{2}^{2}}+\frac{1}{2(1-v)} \frac{\partial^{2} \tilde{u}_{2}}{\partial x_{1} \partial x_{2}}=\frac{s^{2}}{c_{1}^{2}} \tilde{u}_{1} \\
& \frac{1}{2(1-v)} \frac{\partial^{2} \tilde{u}_{1}}{\partial x_{1} \partial x_{2}}+\frac{\partial^{2} \tilde{u}_{2}}{\partial x_{2}^{2}}+\frac{(1-2 v)}{2(1-v)} \frac{\partial^{2} \tilde{u}_{2}}{\partial x_{1}^{2}}=\frac{s^{2}}{c_{1}^{2}} \tilde{u}_{2}
\end{aligned}
$$

Considering the boundary conditions, the nodal displacements can be obtained for certain Laplace parameters $s$ in the Laplace transformed domain.

\section{Hybrid method for crack problems}

The DBEM or DDM are very convenient to analyze crack problems due to their high accuracy and efficiency. However, for nonlinear material properties and geometrically nonlinear problems, the fundamental solutions are not available and therefore, the domain integrals are required. To deal with body forces, the meshless method such as point collocation method is the first option due to its simplicity. Therefore, a hybrid method with the DDM and meshless

method is expected to take advantages of both the DDM and the meshless approach for handling complicated fracture problems.

\subsection{Static case}

We assume that the solutions are combined with

$$
u_{i}=u_{i}^{D}+u_{i}^{M}
$$

for displacements and

$$
\sigma_{i j}=\sigma_{i j}^{D}+\sigma_{i j}^{M}
$$

for stresses, where superscripts " $D$ " and " $M$ " represent the DDM and Meshless respectively. For the DDM in section 2, one has displacements in the domain from Eq.(13) by letting $\theta^{(m)}=0$ 


$$
\begin{aligned}
& u_{1}^{D}(\mathbf{x})=\sum_{l=1}^{N_{c}}\left[U_{1 k}^{(l)}(\mathbf{x}) \cos \left(\theta^{(l)}\right)-U_{2 k}^{(l)}(\mathbf{x}) \sin \left(\theta^{(l)}\right)\right] \psi_{k}^{(l)}=\sum_{l=1}^{N_{c}} U_{1 k}^{(l)}(\mathbf{x}) \psi_{k}^{(l)}, \\
& u_{2}^{D}(\mathbf{x})=\sum_{l=1}^{N_{c}}\left[U_{1 k}^{(l)}(\mathbf{x}) \sin \left(\theta^{(l)}\right)+U_{2 k}^{(l)}(\mathbf{x}) \cos \left(\theta^{(l)}\right)\right] \psi_{k}^{(l)}=\sum_{l=1}^{N_{c}} U_{2 k}^{(l)}(\mathbf{x}) \psi_{k}^{(l)},
\end{aligned}
$$

The stress components $\sigma_{i j}^{D}$ can be obtained from Eq.(13) too. As the DDM solution satisfies the equilibrium equation, displacements $u_{k}^{D}$ disappear in the equilibrium equations Eq.(18) and one has

$$
\begin{aligned}
& \frac{2 \mu(1-v)}{(1-2 v)} \frac{\partial^{2} u_{1}^{M}}{\partial x_{1}^{2}}+\mu \frac{\partial^{2} u_{1}^{M}}{\partial x_{2}^{2}}+\frac{\mu}{1-2 v} \frac{\partial^{2} u_{2}^{M}}{\partial x_{1} \partial x_{2}}+b_{1}=0 \\
& \frac{\mu}{1-2 v} \frac{\partial^{2} u_{1}^{M}}{\partial x_{1} \partial x_{2}}+\frac{2 \mu(1-v)}{(1-2 v)} \frac{\partial^{2} u_{2}^{M}}{\partial x_{2}^{2}}+\mu \frac{\partial^{2} u_{2}^{M}}{\partial x_{1}^{2}}+b_{2}=0
\end{aligned}
$$

Traction boundary conditions in terms of $2 N$ displacement discontinuities $\psi_{k i}$ and meshless nodal values of displacement are given

$$
\begin{aligned}
& \sum_{l=1}^{N_{c}} T_{k}^{n(l m)} \psi_{k}^{(l)}+\sigma_{11}^{M(m)} n_{1}^{2}+\sigma_{22}^{M(m)} n_{2}^{2}+2 \sigma_{12}^{M(m)} n_{1} n_{2}=\bar{t}^{n}\left(\mathbf{x}^{(m)}\right) \\
& \sum_{l=1}^{N_{c}} T_{k}^{\tau(l m)} \psi_{k}^{(l)}+\left(\sigma_{11}^{M(m)}-\sigma_{22}^{M(m)}\right) n_{1} n_{2}+\sigma_{12}^{M(m)}\left(n_{1}^{2}-n_{2}^{2}\right)=\bar{t}^{\tau}\left(\mathbf{x}^{(m)}\right)
\end{aligned}
$$

where $T_{k}^{n(l m)}\left(\mathbf{x}^{(m)}\right)$ and $T_{k}^{\tau(l m)}\left(\mathbf{x}^{(m)}\right)$ are given in Eq.(13) and displacement conditions

$$
\begin{aligned}
& \left.n_{k}\left(u_{k}^{D}+u_{k}^{M}\right)\right|_{\mathbf{x}^{(m)}}=\bar{u}^{n}\left(\mathbf{x}^{(m)}\right) \\
& \left.\tau_{k}\left(u_{k}^{D}+u_{k}^{M}\right)\right|_{\mathbf{x}^{(m)}}=\bar{u}^{\tau}\left(\mathbf{x}^{(m)}\right)
\end{aligned}
$$

where node coordinate $\mathbf{x}^{(m)}$ can collocate on the boundary $\Gamma$ and crack surface $\Gamma_{c}$. Therefore, the unknown number in total is $2 N_{c}+2 N_{m}$, which can be obtained by solving $2 N_{m}$ equations from the meshless approach and $2 N_{c}$ equations from the DDM. It is evident that DDM and meshless method are coupled in the boundary conditions Eqs $(31,32)$ only.

\subsection{Dynamic case}

Similarly to the static case, the solutions of displacement for elastodynamics are coupled as

$$
\tilde{u}_{i}=u_{i}^{D}+\tilde{u}_{i}^{M}
$$


where $u_{i}^{D}$ is given in Eq.(29) with transformed displacement discontinuity $\tilde{\psi}_{k}$ in the Laplace space. Substituting Eq.(33) into the governing equation Eq.(24) results

$$
\begin{aligned}
& \frac{\partial^{2} \tilde{u}_{1}^{M}}{\partial x_{1}^{2}}+\frac{(1-2 v)}{2(1-v)} \frac{\partial^{2} \tilde{u}_{1}^{M}}{\partial x_{2}^{2}}+\frac{1}{2(1-v)} \frac{\partial^{2} \tilde{u}_{2}^{M}}{\partial x_{1} \partial x_{2}}=\frac{s^{2}}{c_{1}^{2}}\left(u_{1}^{D}+\tilde{u}_{1}^{M}\right) \\
& \frac{1}{2(1-v)} \frac{\partial^{2} \tilde{u}_{1}^{M}}{\partial x_{1} \partial x_{2}}+\frac{\partial^{2} \tilde{u}_{2}^{M}}{\partial x_{2}^{2}}+\frac{(1-2 v)}{2(1-v)} \frac{\partial^{2} \tilde{u}_{2}^{M}}{\partial x_{1}^{2}}=\frac{s^{2}}{c_{1}^{2}}\left(u_{2}^{D}+\tilde{u}_{2}^{M}\right)
\end{aligned}
$$

Again, the DDM fundamental solutions of displacement and stress $\left(u_{i}^{D}, \sigma_{i j}^{D}\right)$ are the same for static case with $2 N_{c}$ unknown displacement discontinuities $\psi_{k}^{l}$,

$$
u_{i}^{D}(\mathbf{x})=\sum_{l=1}^{N_{c}} U_{i k}^{(l)}(\mathbf{x}) \tilde{\psi}_{k}^{(l)} \quad \text { and } \quad \sigma_{i j}^{D}(\mathbf{x})=\sum_{l=1}^{N_{c}} T_{i j k}^{(l)}(\mathbf{x}) \tilde{\psi}_{k}^{(l)}
$$

Then, by considering the boundary conditions including crack surface and outer boundary, the nodal displacements of meshless method and displacement discontinuities of the DDM can be obtained for specified Laplace parameter $s$ in the Laplace transformed domain. Suppose a set of sample in the transformation space $s_{g}, g=0,1,2, \ldots, K$, are selected in the Laplace transform domain. Then, the time dependent function can be obtained by the Laplace inversion technique. Here, the algorithm proposed by Durbin [37] is used in this paper as following

$$
f(t)=\frac{2 e^{\eta t}}{T}\left[-\frac{1}{2} \tilde{f}(\eta)+\sum_{k=0}^{K} \operatorname{Re}\left\{\tilde{f}(\eta+2 k \pi i / T) e^{2 k \pi t i / T}\right\}\right]
$$

where $\tilde{f}\left(s_{g}\right)$ represent the transformed variables in the Laplace domain while the Laplace parameters $s_{g}=\eta+2 g \pi i / T \quad(i=\sqrt{-1})$. There are two free parameters $\eta$ and $T$. It is worth to point out that the selection of parameters $T$ depends on the observing period in the time domain and $\eta=5 / T$.

\subsection{Equivalent stress algorithm to determine stress intensity factors}

There are several methods to evaluate stress intensity factors at the crack tip including crack opening displacement (COD), $J$-integrals and weight functions etc. The COD is the simplest way with reasonable degree of accuracy. The $J$-integral and weight function methods are accurate but their computational efforts are much larger due to domain/boundary integrals. An accurate algorithm by the DDM is called as the equivalent stress technique proposed by Wen 
$[22,23]$ in both static and dynamic cases. It is clear that the discontinuity displacements $\psi_{k}^{(l)}$ on the crack elements are the true opening displacements between the upper and lower crack surfaces; therefore, the stress intensity factors are determined by $\psi_{k}^{(l)}$ only. Suppose the crack is a straight line (or part of crack in front of crack tip) which is divided into $N_{\text {inc }}$ segments as shown in Figure 4. In the local coordinate system, one has

$$
x_{1}^{l}=\left(\frac{2 l-1}{N_{i n c}}-1\right) a_{f} \text { and } \Delta^{(l)}=\frac{a_{f}}{N_{i n c}}
$$

where $a_{f}$ is the half-length of crack extension ( $a_{f}=a_{0}$ for initial crack length). The equivalent normal and shear stresses $\bar{\sigma}^{(m)}$ and $\bar{\tau}^{(m)}$ [20] can be obtained from the solutions of the discontinuity displacement in Eq.(9) as

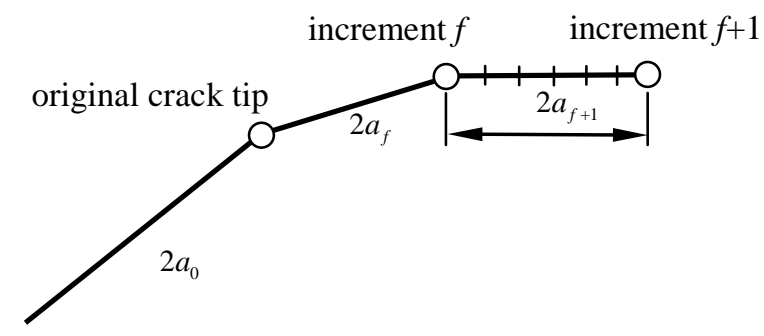

Figure 4. Crack growth by straight line segments at crack tips.

$$
\bar{\sigma}^{(m)}=\sum_{l=1}^{N_{\text {inc }}} \alpha_{m l} \psi_{2}^{(l)}, \bar{\tau}^{(m)}=\sum_{l=1}^{N_{\text {inc }}} \alpha_{m l} \psi_{1}^{(l)}
$$

where the coefficient

$$
\alpha_{m l}=\frac{\mu \Delta^{(l)}}{\pi(1-v)\left[\left(x_{1}^{(l)}-x_{1}^{(m)}\right)^{2}-\Delta^{(l) 2}\right]}
$$

in which $\Delta^{(l)}$ is the half-length of segment $l$, and $x_{1}^{(l)}$ is the mid-point of segment $l$ in the local coordinate shown in Figure 2(a). From the stress intensity factor handbook [38], one has

$$
K_{I}^{ \pm}=\frac{1}{\sqrt{\pi a_{f}}} \int_{-a_{f}}^{a_{f}} \bar{\sigma}\left(x_{1}\right) \sqrt{\frac{a_{f} \pm x_{1}}{a_{f} \mp x_{1}}} d x_{1}=\sum_{m=1}^{N_{\text {inc }}} \sum_{l=1}^{N_{\text {inc }}} F^{ \pm m} \alpha_{m l} \psi_{2}^{(l)} \sqrt{\pi a_{f}}
$$




$$
K_{I I}^{ \pm}=\frac{1}{\sqrt{\pi a_{f}}} \int_{-a_{f}}^{a_{f}} \bar{\tau}\left(x_{1}\right) \sqrt{\frac{a_{f} \pm x_{1}}{a_{f} \mp x_{1}}} d x_{1}=\sum_{m=1}^{N_{\text {inc }}} \sum_{l=1}^{N_{\text {inc }}} F^{ \pm m} \alpha_{m l} \psi_{1}^{(l)} \sqrt{\pi a_{f}}
$$

where the coefficient

$$
F^{ \pm m}=\frac{1}{\pi a_{f}} \int_{x_{1}^{(m)}-\Delta^{(m)}}^{x_{1}^{(m)}+\Delta^{(m)}} \sqrt{\frac{a_{f} \pm x_{1}}{a_{f} \mp x_{1}}} d x_{1}=\frac{1}{\pi}\left[\arcsin \left(\frac{x_{1}}{a}\right) \pm \sqrt{1-\left(\frac{x_{1}}{a}\right)^{2}}\right]_{x_{1}^{(m)}-\Delta^{(m)}}^{x_{1}^{(m)}+\Delta^{(m)}}
$$

The positive sign refers to the right-hand crack tip and the negative sign to the left-hand tip. From Eq.(40), it is obvious that the singularity of stresses depend on the discontinuity displacements of all the crack segments. However, the closer the segment is to the crack tip, the greater its influence. In addition, the dynamic stress intensity factors in the Laplace domain can be written in the same form as the statics by replacing the static displacement discontinuities $\psi_{k}^{l}$ in Eq.(40) with the transformed one $\tilde{\psi}_{k}^{l}$.

\subsection{Crack growth}

The crack propagation path is a smooth curve. However, crack propagation is simulated by successive linear increments in numerical approach. Several criteria have been proposed to describe the direction of crack growth. The maximum principal stress criterion is more popular in engineering and is adopted in this paper. This criterion postulates that the growth of crack will occur in a direction perpendicular to the maximum principal stress. Thus, at each new crack tip, the local direction of crack growth is determined by the condition that the singular shear stress is zero, that is

$$
K_{\mathrm{I}} \sin \theta_{c}+K_{\mathrm{II}}\left(3 \cos \theta_{c}-1\right)=0
$$

and therefore

$$
\theta_{c}= \begin{cases}2 \arctan \left[\left(\varphi-\sqrt{\varphi^{2}+8}\right) / 4\right] & \text { for } K_{I I}>0 \\ 2 \arctan \left[\left(\varphi+\sqrt{\varphi^{2}+8}\right) / 4\right] & \text { for } K_{I I}<0\end{cases}
$$

where $\varphi=K_{I} / K_{I I}$. The computation procedure can be summarized as following

(i) Input of data for meshless and DDM including geometry and nodes distribution, crack segments and boundary conditions; 
(ii) Assemble system of equations and use linear equations solver;

(iii) Determine mixed-mode stress intensity factors and crack growth angle;

(iv) Model crack propagation with $N_{\text {inc }}$ segments on one crack extension;

(v) Update/output crack path;

(vi) Go to (ii) if necessary for next crack extension, otherwise stop.

\section{Numerical examples}

\subsection{A rectangular plate with a central crack}

In order to validate the applicability of the MDDM proposed in this paper, a rectangular plate containing a central crack is considered as shown in Figure 5 loaded by a uniform tensile stress $\sigma_{0}$ on the top and bottom of the plate. Firstly, the regularly distributed nodes $\left(N_{1} \times N_{2}\right)$ are specified in the domain for the meshless method and segments $\left(N_{c}\right)$ for the DDM are used on the crack surface. The Poisson ratio $v$ is taken as 0.3 with plane strain assumption. Densities of nodes and segment $N_{1}=N_{2}=N_{c}=N=20$. The radius of the support domain $r_{\eta}$ centered at collocation $\boldsymbol{\eta}$ is determined such that the minimum number of nodes in the support domain $L_{m}=12$ and the shape parameter in the multiquadric radial basis function $c=8 r_{m}$. By computational tests, the numerical solutions are stable with a large range selection of parameter c. The relative error $\left(\varepsilon(\%)=100 \times\left|K_{I}^{M D D M}-K_{I}^{*}\right| / K_{I}^{*}\right)$ versus the node number $N$ are shown in Table 2 to demonstrate the convergence and stability when $a / b=0.5$, where the reference value is from the handbook [38] $K_{I}^{*}=1.9897 \sigma_{0} \sqrt{\pi a}$. Excellent agreement can be obtained when $N \geq 20$. To demonstrate the convergence of the free parameter in the multi-quadrics, the relative errors are also shown in Figure 6 against the dimensionless number $\varsigma, c=\varsigma r_{\eta}$ while $N=20$. It can be seen that the accurate results can be obtained in the region of $4 \leq \varsigma \leq 14$ with the relative error less than $4 \%$. The stress intensity factors $K_{I}$ versus the ratios $h / b$ and $a / b$ are plotted in Figure 7. Compared with the existing results in handbook [38], good agreement is achieved. 
Table 2. Convergence study

\begin{tabular}{ccc}
\hline$N$ & MDDM & $\varepsilon(\%)$ \\
\hline 10 & 1.8292 & 8.1 \\
20 & 2.0604 & 3.6 \\
30 & 2.0587 & 3.5 \\
40 & 2.0371 & 2.4 \\
Ref.[38] & 1.9897 & --- \\
\hline
\end{tabular}

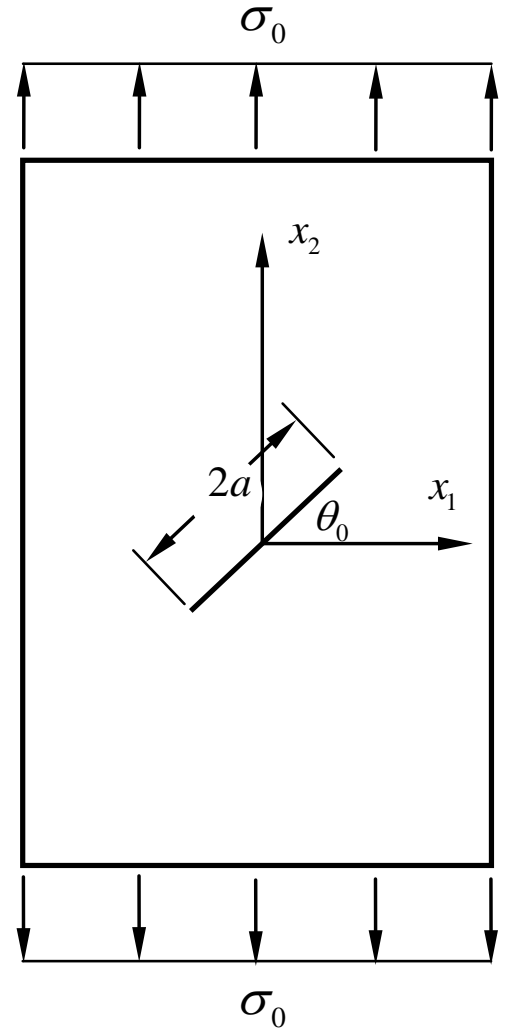

Figure 5. Rectangular plate with a central slant crack of length $2 a$. 


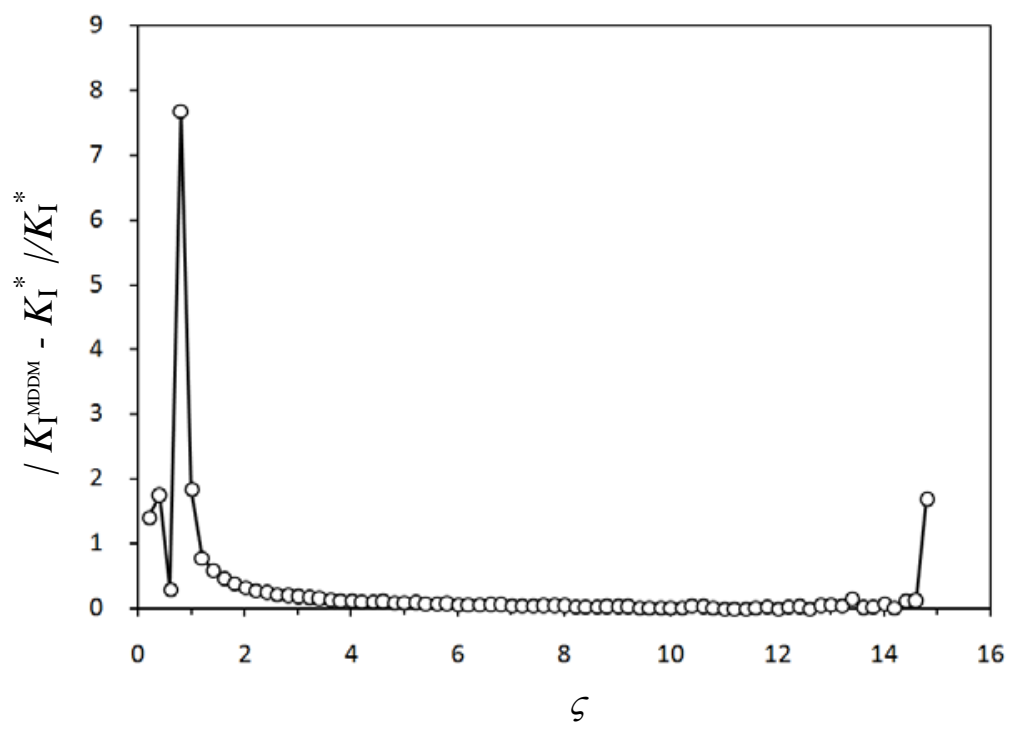

Figure 6. The influence of dimensionless parameter $\varsigma$ in multiquadric RBF.

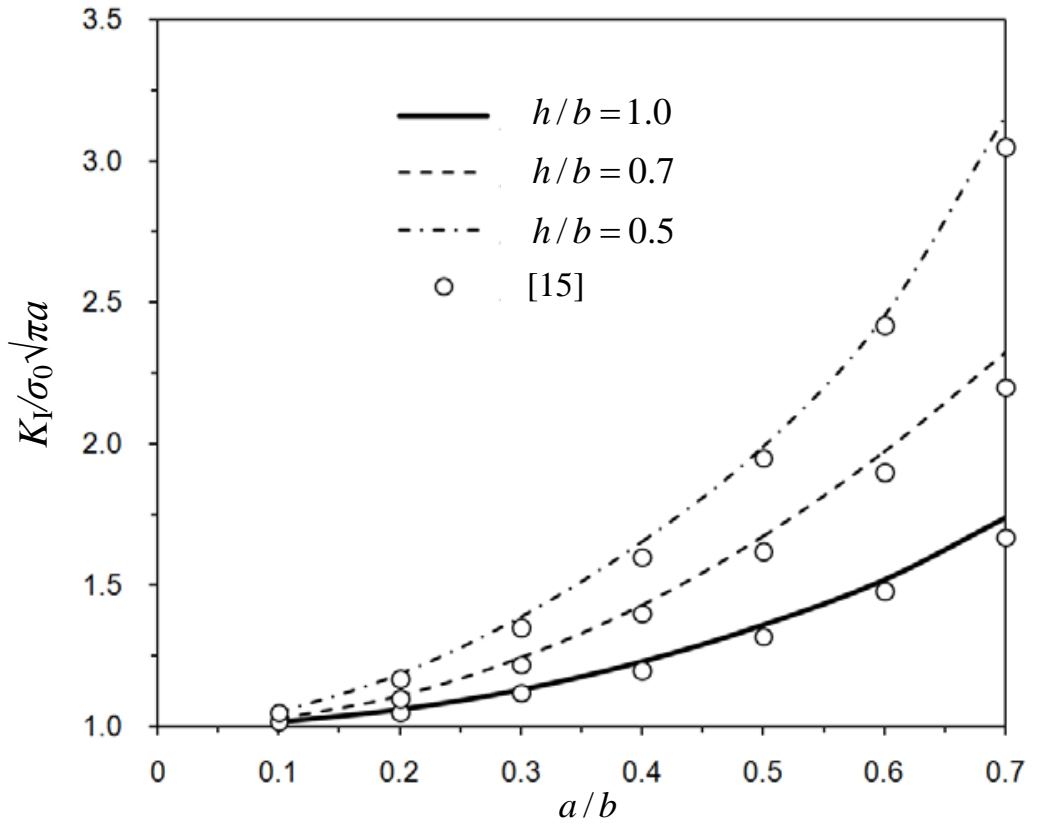

Figure 7. The influence of free parameter c in multiquadric RBF. 


\subsection{A circular plate with a central crack}

A circular plate of radius $R$ with an embedded crack length $2 a$ subjected to either a uniform load shown in Figure 8(a) on the boundary or rotation with angle speed $\omega$ in Figure 8(b). A plane strain assumption is considered and mass density $\rho$. The distribution of nodes using the meshless approach is shown in Figure 8(c) with 335 nodes in total and the crack segment number $N_{c}=20$ in the DDM. For the case of rotation, the body forces can be written as $b_{k}=\rho \omega^{2} x_{k}(k=1,2)$. Normalized stress intensity factors for uniform tensile $K_{I} / \sigma_{0} \sqrt{\pi a}$ and for rotation $K_{I} / \rho \omega^{2} R^{2} \sqrt{\pi a}$ with different Poisson ratios are presented in Figures 9 and 10 respectively. Also the analytical solutions from [38] are presented in the same figure in order to show the accuracy of the MDDM.

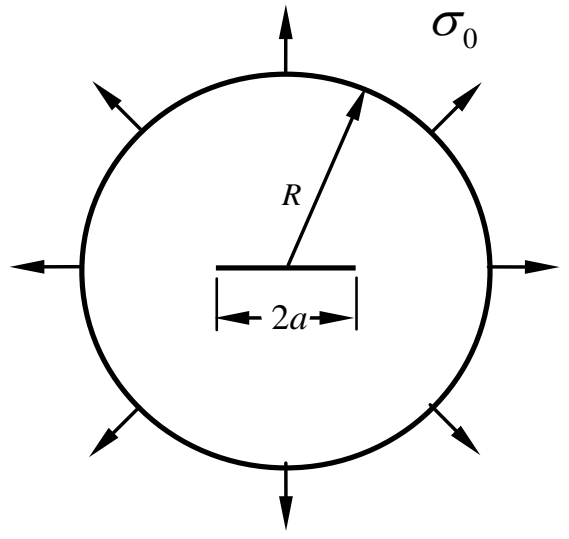

(a)

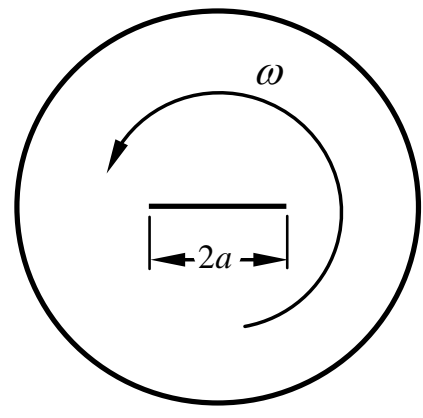

(b)

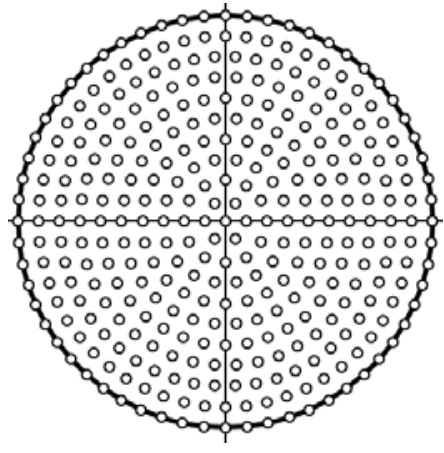

(c)

Figure 8. Circular plate with central crack under (a) tensile load, (b) rotation around centre of plate and (c) node distribution in MDDM. 


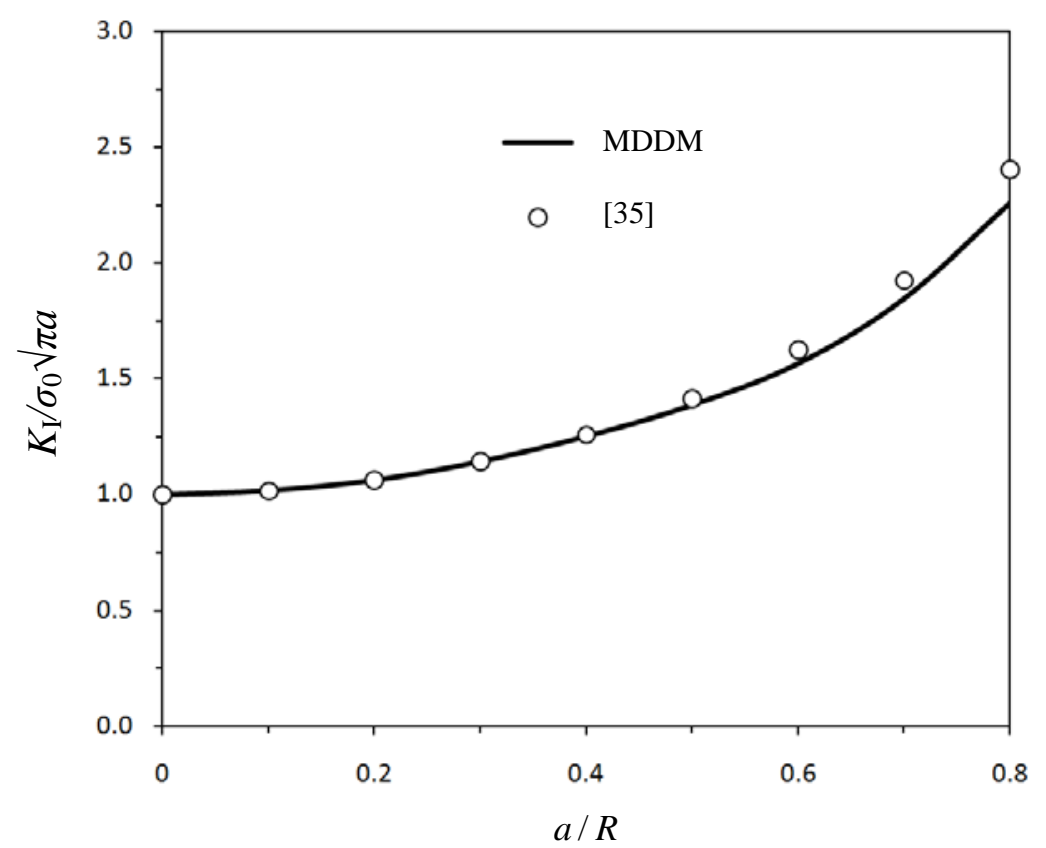

Figure 9. Normalized stress intensity factors under uniform tension $\sigma_{0}$.

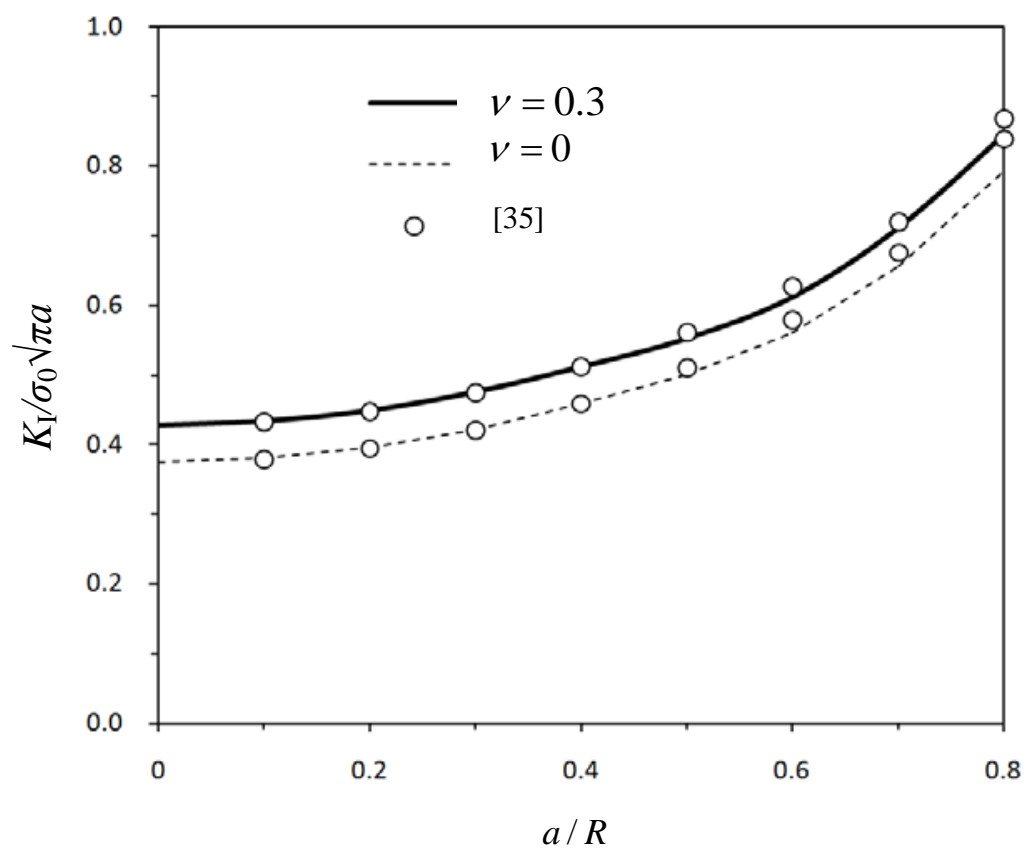

Figure 10. Normalized stress intensity factors with angle speed $\rho$. 


\subsection{Fatigue crack growth for a single edge slant crack}

In linear elasticity, the conventional crack growth approach is based on the singular stress portions at the crack tip represented by mixed mode stress intensity factors in general case. In this paper, a rectangular plate of width $2 b$ and height $2 h$ containing an inclined central crack of length $2 a_{0}$ with angle $\theta=45^{\circ}$ is considered first. We assume the ratio $h / b=1$, the density of nodal distribution $N_{1}=N_{2}=20$ and segment number $N_{c}=100$ for initial crack. Again, the minimum number of node in the support domain $L_{m}=12$ and the dimensionless shape parameter in the multiquadric radial basis function $\varsigma=8$. For each crack growth, the half pace of extension is taken as $a_{f}=\Delta a=0.05 a_{0}$ and the number of segment $N_{i n c}=10$ in each extension. The crack propagations are shown in Figure 11 for different initial crack lengths $a_{0}$. Obviously the angle of crack extension is always negative and tends to zero gradually. Normalized stress intensity factors $K_{I, I I} / \sigma \sqrt{\pi a_{0}}$ versus the crack extension measured from crack tip $s=2 \sum a_{f}$ are shown in Figure 12. Noticeably, there is a jump for both mode I and mode II stress intensity factors at the first crack growth. The mode II stress intensity factor drops to zero immediately. The FEM results by Patrício et al [42] are plotted in Figure 13 for compression. A good agreement can be seen at the beginning of crack propagation. However, the gap is increased gradually when crack tip coordinate $x_{1}>0.3$. It is also observed that using the FEM, the angle of crack growth $\theta_{c}$ becomes positive, which is not reasonable. Similar case is analyzed by Andrade and Leonel [40] by using the DBEM and the crack growth angle $\theta_{c}$ is always negative and tends to zero gradually as expected. 


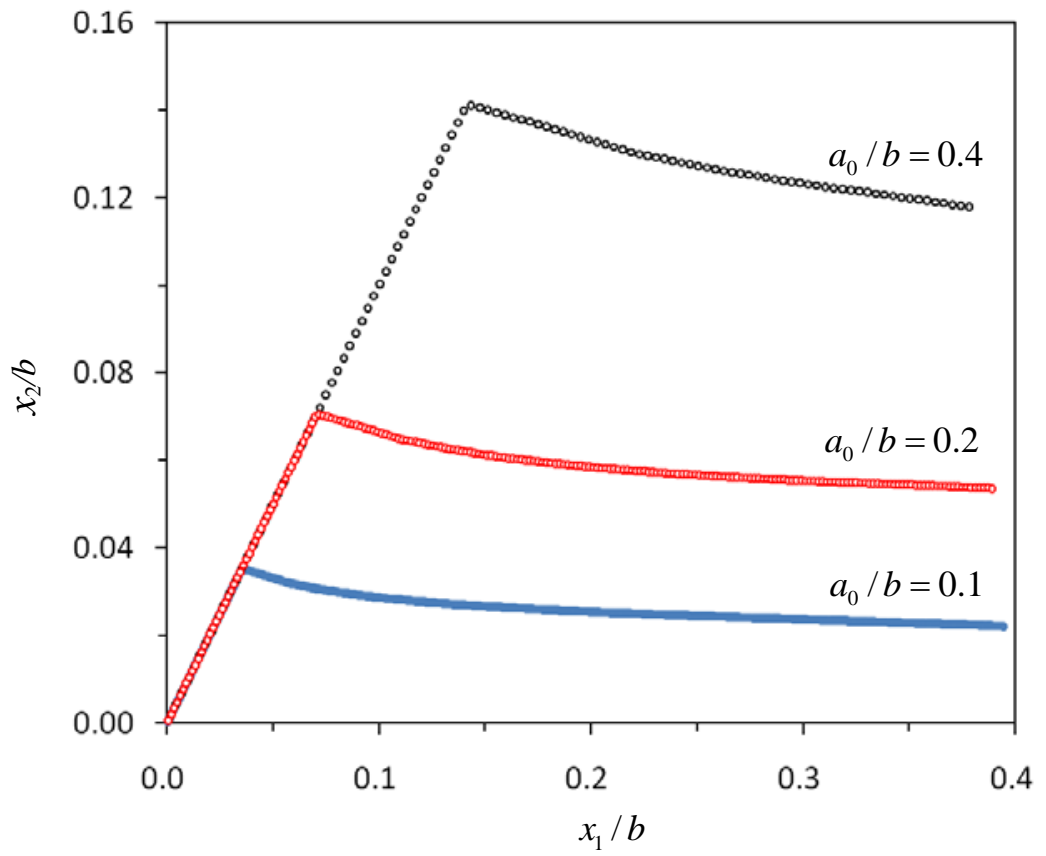

Figure 11. Crack growth path with different initial crack lengths $a_{0}$.

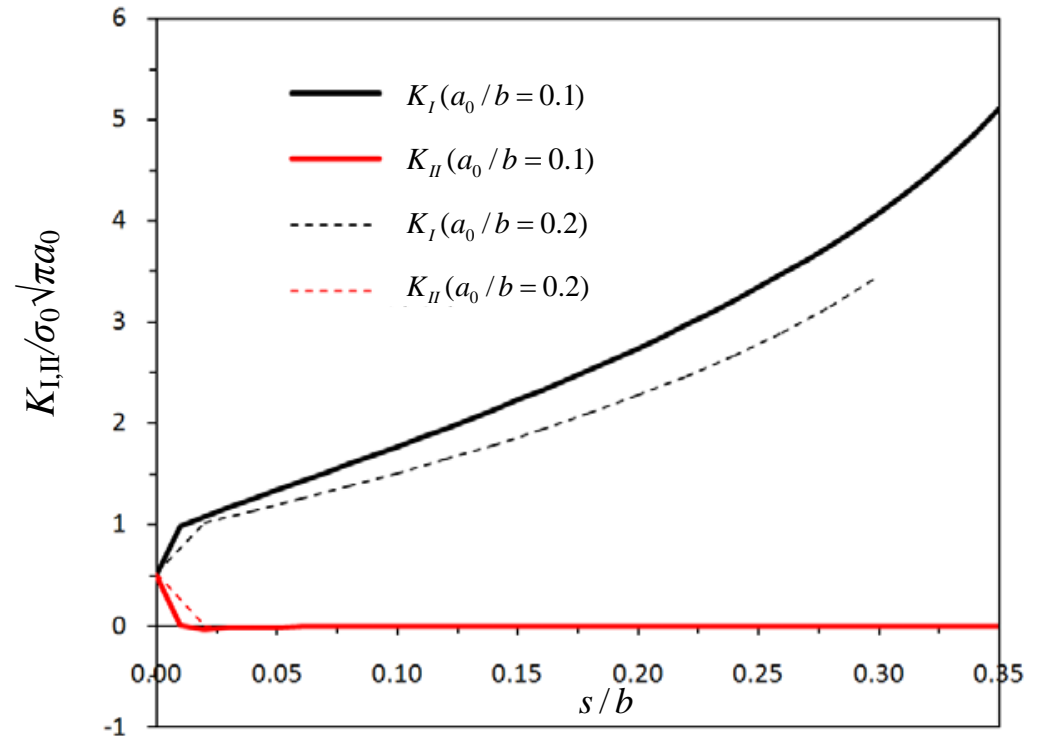

Figure 12. Variation of normalized stress intensity factors against the crack extension $s$, where $s=2 \sum a_{f}$. 


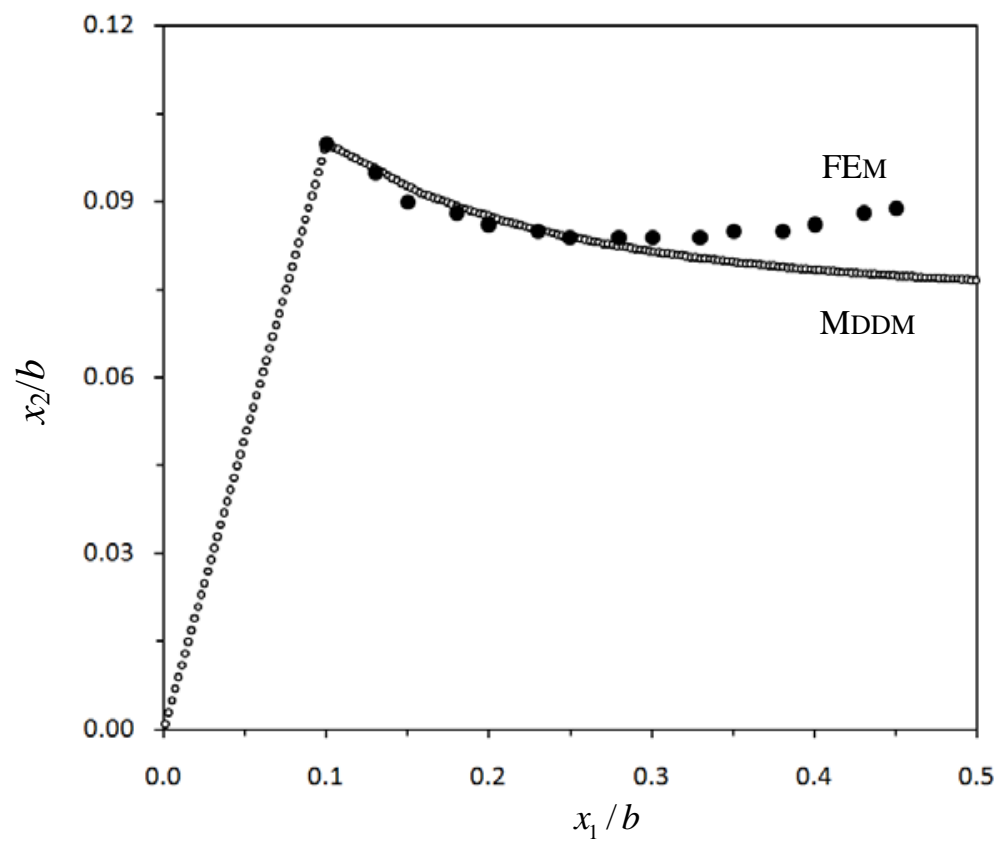

Figure 13. Crack growth pathes by MDDM and FEM.

\subsection{Rectangular plate with one central crack under dynamic load}

For dynamic loadings, we consider a rectangular plate of width $2 b$ and length $2 h$ with a centrally located crack of length $2 a$, firstly, as shown in Figure 5, when $\theta=0$. It is loaded in the direction perpendicular to the crack by a uniform tension $\sigma_{0} H(t)$, where $H(t)$ is the Heaviside function with dimensions $h / b=2, a / b=0.24, h=20 \mathrm{~cm}$ with material properties: shear modulus $\mu=0.76923 \mathrm{Mb}$; density $\rho=5 \mathrm{~g} / \mathrm{cm}^{3}$ and $\nu=0.3$ (plane strain state). It was studied firstly by Chen in [41]. Therefore, we have elastic wave speeds $c_{1}=0.743 \mathrm{~cm} / \mu \mathrm{sec}$, $c_{2}=0.392 \mathrm{~cm} / \mu \mathrm{sec}$ (shear wave speed) and $c_{R}=0.363 \mathrm{~cm} / \mu \mathrm{sec}$ (surface wave speed). Let the number of sample in Laplace space $K=25$, time $T=10 t_{0}, t_{0}=h / c_{1}$ and $\eta=5 / T$. All free parameters are kept the same as in previous example 5.1. In order to validate the convergence of MDDM. Firstly, two nodal densities are considered, i.e. $N_{1}=10, N_{2}=20, N_{c}=10$ and $N_{1}=20, N_{2}=40, N_{c}=20$. The variations of the dynamic stress intensity factors 
$K_{I}(t) / \sigma_{0} \sqrt{\pi a}$ are shown in Figure 14. Clearly the differences between the two dynamic stress intensity factors with two nodal distributions are reasonably small. Comparison between difference results by Wen [42], Chen [41], Fedelinski et al [43] and are also shown in Figure 15. The first sharp peak can be observed clearly by the MDDM which is caused by the elastic surface wave. There are two free parameters in the Durbin's Laplace inverse method, i.e. $\eta$ and $T$. The stability and accuracy analysis influenced by these two parameters were studied comprehensively in by Durbin [37] for general case and by Wen et al [22,23] for solid mechanics.

Finally, a rectangular plate with a central slant crack is observed with the same material and configurations above. The angle of the crack $\theta=45^{\circ}$ as in Figure 5. The normalized mixed dynamic stress intensity factors $K_{I}(t) / \sigma_{0} \sqrt{\pi a}$ and $K_{I I}(t) / \sigma_{0} \sqrt{\pi a}$ are shown in Figure 16 for two crack lengths (dash lines for the case of $a / b=\sqrt{2} / 3$ and solid lines for $a / b=\sqrt{2} / 2$ ). Compared with the BEM [42], excellent agreement is achieved.

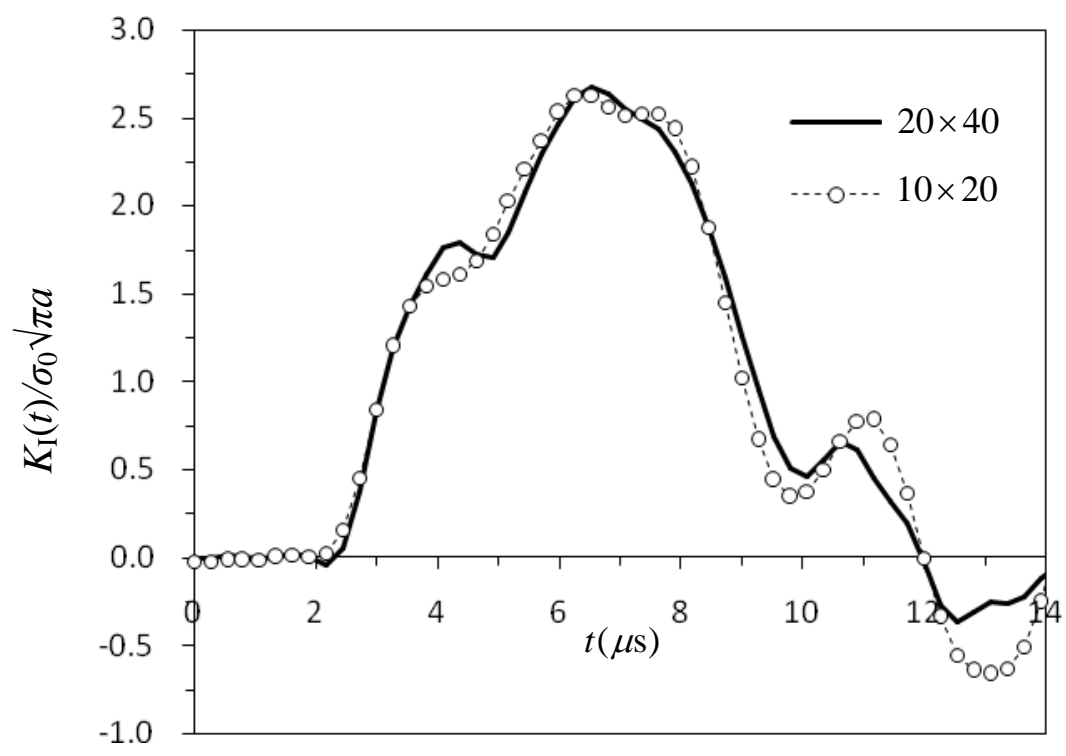

Figure 14. Normalized dynamic stress intensity factor $K_{I}(t) / \sigma_{0} \sqrt{\pi a}$ for different nodal densities. 


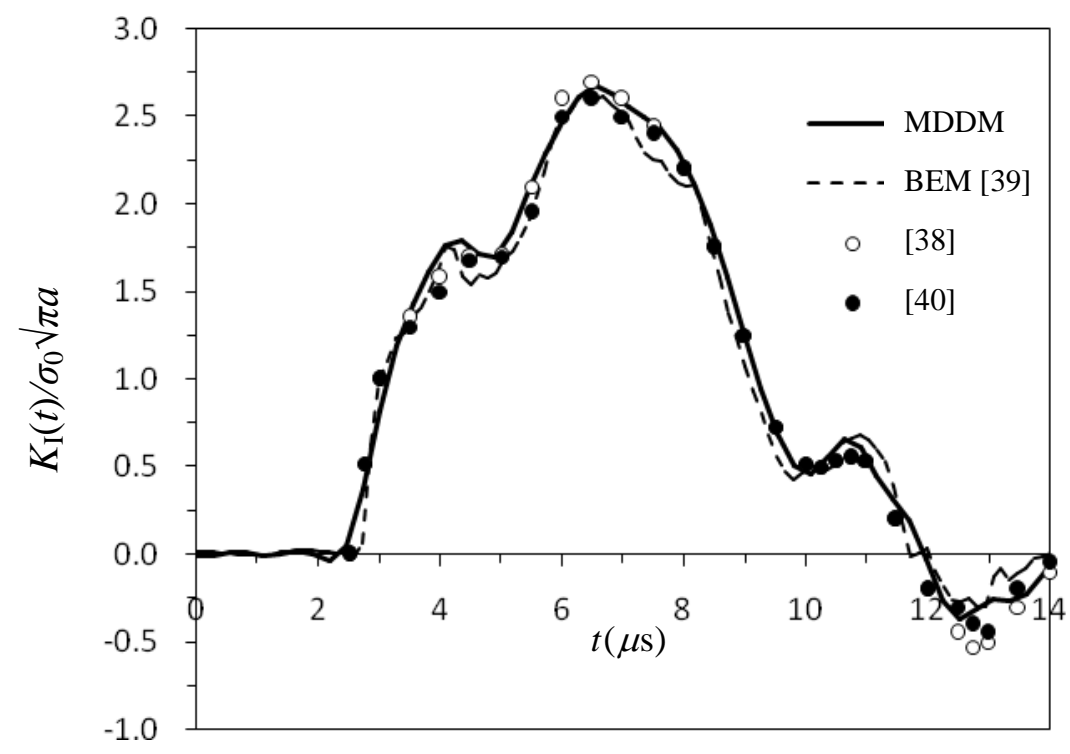

Figure 15. Normalized dynamic stress intensity factor $K_{I}(t) / \sigma_{0} \sqrt{\pi a}$ with different approaches.

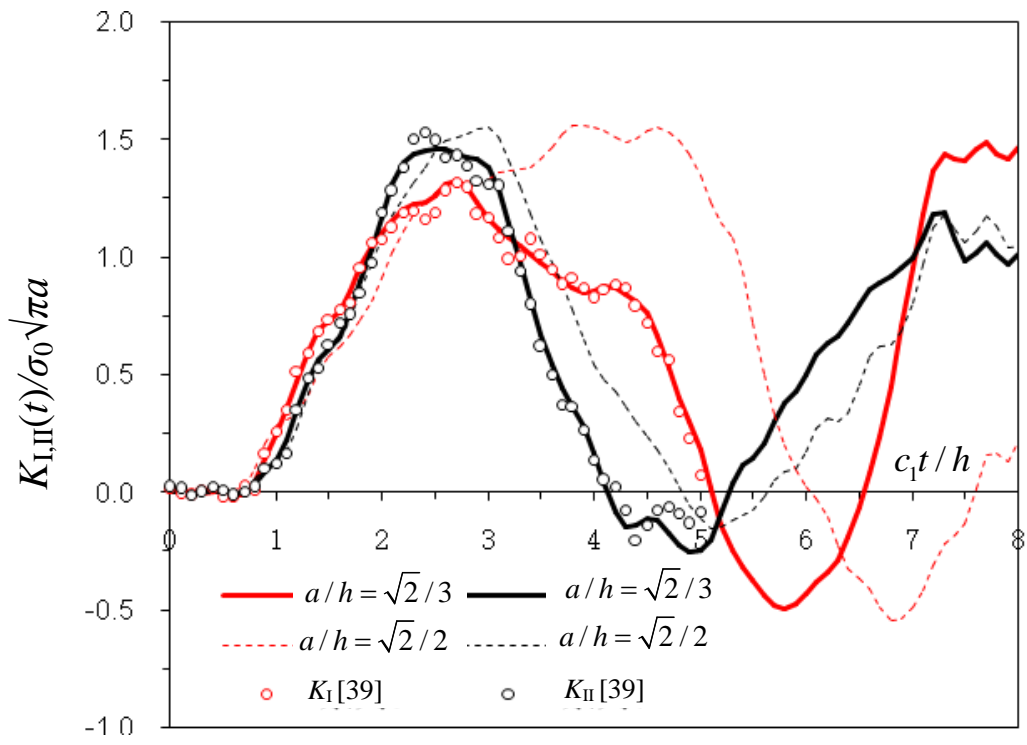

Figure 16. Normalized dynamic stress intensity factors $K_{I, I I}(t) / \sigma_{0} \sqrt{\pi a}$ versus the normalized time $c_{1} t / h$ with different approaches. 


\section{Conclusion}

This paper presented a hybrid method with meshless and displacement discontinuity procedure for two-dimensional fracture mechanics under static and dynamic loads. By BEM including DBEM, the fundamental solutions have to be derived both for static and dynamic problems. I addition, the fundamental solutions are complicated either in the time domain or in Laplace transformed domain. The main advantage of MDDM is that the static fundamental solutions can be adopted to deal dynamic case directly. Advantages of meshless method and displacement discontinuity method are inherited to deal with fracture problems more efficiently. The MDDM is of high accuracy with body forces (Meshless) and mixed-mode crack propagation problem (DDM). The static fundamental solutions of DDM are required only for cases of dynamics. Although this paper analyses the crack growth for two dimensional isotropic material only, the MDDM can be extended to anisotropic cracked structures with functionally graded materials and other nonlinear phenomenon.

\section{Acknowledgment.}

The authors acknowledge the supports of the Slovak Science and Technology Assistance Agency registered under number APVV-18-0004, VEGA-2/0061/20, Hunan Provincial Natural Science Foundation of China (Grant No. 2018JJ3519) and Scientific Research Project of Hunan Provincial Office of Education (Grant no. 17B003).

\section{References}

[1] O. Kolednik, J. Predan, F.D. Fischer, Cracks in inhomogeneous materials: comprehensive assessment using the configurational forces concept, Eng Fract Mech, 77 (2010), 3611-3624.

[2] O. Kolednik, J. Zechner, J. Predan, Improvement of fatigue life by compliant and soft inter layers, Scr. Mater., 113 (2016), 1-5.

[3] B. Wang, T. Siegmund, Simulation of fatigue crack growth at plastically mismatched bimaterial interfaces, Int. J. Plast., 22 (2006), 1586-1609. 
[4] R.H. Gallagher, A review of finite element techniques in fracture mechanics, Proc. of the first conference on numerical methods in fracture mechanics, A.R. Luxmoore and D.R.J. Owen edited, University college of Swansea, 1-25, 1978.

[5] J.R. Rice, D.M. Tracey, Computational fracture mechanics, in numerical and computer methods in structural mechanics, Academia Press, New York, 1973.

[6] M.S. Shephard, N.A.B. Yehia, G.S. Burd, T.J. Weidner, Automatic crack propagation tracking, Comp. and Struct., 20 (1985), 211-223.

[7] J.R. Rice, A path independent integral and the approximate analysis of strain concentration by notches and cracks, J. Applied Mechanics, Transaction of ASME, 35(1968), 379-386.

[8] H. Yu, L. Wu, L. Guo, S. Du, Q. He, Investigation of mixed-mode stress intensity factors for nonhomogeneous materials using an interaction integral method, Int. J. Solids Struct., 46 (2009), 3710-3724.

[9] S. Kumar, V. Singh B.K. Mishra, A homogenized XFEM approach to simulate fatigue crack growth problems, Computers and Structures, 150 (2015), 1-22.

[10] B. Wang, H. De Backer, A. Chen, An XFEM based uncertainty study on crack growth in welded joints with defects, Theor. Appl. Fract. Mech., 86 (2016), 125-142.

[11] A.R. Ingraffea, G.E. Blandford, J.A. Ligget, Automatic modelling of mixed- mode fatigue and quasi-static crack propagation using the boundary element method, Proc. Fracture Mechanics, 14th Symposium, ASTM STP 791(1983), 407-426, ASTM.

[12] W.H. Grestle, Finite and boundary element modelling of crack propagation in two- and three-dimensions using interactive computer graphics, PhD Thesis, Cornell University, Ithaca, USA, 1986.

[13] Z Cen and G. Maier, Bifurcation and instabilities in fracture of cohesive-softening structures: a boundary element analysis, Fatigue and Fracture of Engineering Materials and Structures, 15(1992), 911-928.

[14] A. Portela, M.H. Aliabadi, D.P. Rooke, Dual boundary incremental analysis of crack propagation, Computer and Structures, 46 (2)(1993), 237-247.

[15] Y. Mi and M.H. Aliabadi, Three-dimensional crack growth simulation using BEM, Computers and Structures, 52(1994), 871-878.

[16] Y. Mi and M.H. Aliabadi, An automatic procedure for mixed-mode crack growth analysis, Communications in Numerical Methods, 33(1995), 167-177 
[17] M.H. Aliabadi, The boundary element method: Applications in solids and structures, John Wiley \& Sons, Ltd., West Sussex, England, 2002.

[18] S.L. Crouch and A.M. Starfield, Boundary Element Methods in Solid Mechanics, George Allen and Unwin, London, 1983.,

[19] P.H. Wen, The calculating stress intensity factor for a rigid wedge inserted into a halfinfinite crack in an orthotropic plate, Chinese J. Applied Mech., 5 (4)(1988), 89-96.

[20] P.H. Wen, The solution of a displacement discontinuity for an anisotropic half-plane and its applications to fracture mechanics, Eng. Fract. Mech., 35 (5/6)(1989), 1145-1154.

[21] P.H. Wen, The calculation of SIF considering the effects of arc crack surface contact and friction under uniaxial tension and pressure, Eng. Fract. Mech., 36 (4)(1991), 651-660.

[22] P.H. Wen, M.H. Aliabadi \& D.P. Rooke, The influence of elastic waves on dynamic stress intensity factors (three dimensional problem), Archive of Applied Mechanics, 66(6)(1996), 385384.

[23] P.H. Wen, M.H. Aliabadi \& D.P. Rooke, The influence of elastic waves on dynamic stress intensity factors (two dimensional problem), Archive of Applied Mechanics, 66(5)(1996), 326335.

[24] R.L. Hardy. Multiquadric equations of Topography and other irregular surfaces. Journal of Geophysical Research, 176(1971),1905-1915.

[25] T. Belytschko, Y.Y. Lu, L. Gu, Element-free Galerkin method. Int. J. Numerical Methods in Engineering, 37(1994), 229-256.

[26] H. Pathak, A. Singh, I.V. Singh, Fatigue crack growth simulations of homogeneous and bimaterial interfacial cracks using element free Galerkin method, Appl. Math. Model., 38 (2014), 3093-3123.

[27] A. Jameel, G.A. Harmain, Fatigue crack growth in presence of material discontinuities by EFGM, Int. J. Fatigue, 81 (2015), 105-116.

[28] N. Muthu, S.K. Maiti, B.G. Falzon, W. Yan, Crack propagation in non-homogenous materials: evaluation of mixed-mode SIFs, T-stress and kinking angle using a variant of EFG Method, Eng. Anal. Bound. Elem., 72 (2016), 11-26.

[29] Y.C. Hon, X.Z. Mao, A multiquadric interpolation method for solving initial value problems, J. Scientific Computing, 12(1997), 51-55. 
[30] S.N. Atluri, The Meshless Method (MLPG) for Domain and BIE Discretizations, Forsyth, GA, USA, Tech Science Press, 2004.

[31] V. Sladek, J. Sladek, M. Tanaka, Ch. Zhang, Local integral equation method for potential problems in functionally graded anisotropic materials, Engng Analy. with Boundary Elements, 29(2005), 829-843.

[32] J. Sladek, V. Sladek, P.H. Wen, M.H. Aliabadi, Meshless Local Petrov-Galerkin (MLPG) method for shear deformable shells analysis. CMES: Computer Modeling in Engineering \& Sciences 13(2006), 103-117.

[33] P.H. Wen, M.H. Aliabadi, Crack growth modelling in functionally graded materials by mesh-free method, SDHM Structural Durability and Health Monitoring, 8 (3)(2012), 223-247.

[34] Z.J. Fu, Q. Xi, W. Chen, A.H.D. Cheng, A boundary-type meshless solver for transient heat conduction analysis of slender functionally graded materials with exponential variations. Computers \& Mathematics with Applications, 76(4) (2018), 760-773.

[35] Z.J. Fu, W. Chen, H.T. Yang, Boundary particle method for Laplace transformed time fractional diffusion equations. Journal of Computational Physics, 235(2013), 52-66.

[36] Z.J. Fu, L.W. Yang, H.Q. Zhu, W.Z. Xu, A semi-analytical collocation Trefftz scheme for solving multi-term time fractional diffusion-wave equations. Engineering Analysis with Boundary Elements, 98(2019), 137-146.

[37] F. Durbin, Numerical inversion of Laplace transforms: an efficient improvement to Dubner and Abate's method, The Computer J., 17(1975), 371-376.

[38] D.P. Rooke, D.J. Cartwright, A Compendium of Stress Intensity Factors, HMSO, London, 1976.

[39] M. Patrício, R.M.M. Mattheij, Crack propagation analysis (CASA-report; Vol. 0723). Eindhoven: Technische Universiteit Eindhoven, 2007.

[40] H. D. C. Andrade and E.D. Leonel, The multiple fatigue crack propagation modelling in nonhomogeneous structures using the DBEM, Eng. Analy. with Boundary Elements, 98(2019), 296-309.

[41] Y.M. Chen, Numerical computation of dynamic stress intensity factors by a Lagrangian finite-difference method (the HEMP CODE), Eng. Fracture Mech., 7(1975), 653-660.

[42] P.H. Wen, Dynamic Fracture Mechanics: Displacement Discontinuity Method, Computational Mechanics Publications, Southampton UK and Boston USA, 1996. 
[43] P. Fedelinski, M.H. Aliabadi, D.P. Rooke, The dual boundary element method: J-integral for dynamic stress intensity factors, Int. J. Fracture, 65(1994), 369-381.

\section{Appendix A. Partial differentials of function $f$}

From Eqs.(10a), (10b), (11a) and (11b), the partial differentials for different order are obtained by

$$
\begin{aligned}
& f_{, 1}=\frac{1}{4 \pi(1-v)} \ln \sqrt{\frac{\left(x_{1}-\Delta\right)^{2}+x_{2}^{2}}{\left(x_{1}+\Delta\right)^{2}+x_{2}^{2}}} \\
& f_{, 2}=-\frac{1}{4 \pi(1-v)}\left(\arctan \left(\frac{x_{2}}{x_{1}-\Delta}\right)-\arctan \left(\frac{x_{2}}{x_{1}+\Delta}\right)\right), \\
& f_{, 11}=-\frac{1}{4 \pi(1-v)}\left(\frac{x_{1}-\Delta}{\left(x_{1}-\Delta\right)^{2}+x_{2}^{2}}-\frac{x_{1}+\Delta}{\left(x_{1}+\Delta\right)^{2}+x_{2}^{2}}\right), f_{, 22}=f_{, 11}, \\
& f_{, 12}=\frac{x_{2}}{4 \pi(1-v)}\left(\frac{1}{\left(x_{1}-\Delta\right)^{2}+x_{2}^{2}}-\frac{1}{\left(x_{1}+\Delta\right)^{2}+x_{2}^{2}}\right), \\
& f_{, 111}=-\frac{1}{4 \pi(1-v)}\left(\frac{\left(x_{1}-\Delta\right)^{2}-x_{2}^{2}}{\left[\left(x_{1}-\Delta\right)^{2}+x_{2}^{2}\right]^{2}}-\frac{\left(x_{1}+\Delta\right)^{2}-x_{2}^{2}}{\left[\left(x_{1}+\Delta\right)^{2}+x_{2}^{2}\right]^{2}}\right), f_{, 122}=-f_{, 111}, \\
& f_{, 222}=\frac{x_{2}}{2 \pi(1-v)}\left(\frac{x_{1}-\Delta}{\left[\left(x_{1}-\Delta\right)^{2}+x_{2}^{2}\right]^{2}}-\frac{x_{1}+\Delta}{\left[\left(x_{1}+\Delta\right)^{2}+x_{2}^{2}\right]^{2}}\right), \quad f_{, 112}=-f_{, 222} .
\end{aligned}
$$

